\title{
Dynamical overlap fermion simulations with a preconditioned Hybrid Monte Carlo force
}

\author{
Jan Volkholz* and Wolfgang Bietenholz \\ Institut für Physik, Humboldt-Universität zu Berlin \\ Newtonstr. 15, D-12489 Berlin, Germany \\ E-mail: volkholz@physik.hu-berlin.de, bietenho@physik.hu-berlin.de
}

\section{Stanislav Shcheredin}

Fakultät für Physik, Universität Bielefeld

D-33615 Bielefeld, Germany

E-mail: shchered@physik.hu-berlin.de

\begin{abstract}
We present simulation results for the 2-flavour Schwinger model with dynamical Ginsparg-Wilson fermions. Our Dirac operator is constructed by inserting an approximately chiral hypercube operator into the overlap formula, which yields the overlap hypercube operator. Due to the similarity with the hypercubic kernel, a low polynomial of this kernel can be used as a numerically cheap way to evaluate the fermionic part of the Hybrid Monte Carlo force. We verify algorithmic requirements like area conservation and reversibility, and we discuss the viability of this approach in view of the acceptance rate. Next we confirm a high level of locality for this formulation. Finally we evaluate the chiral condensate at light fermion masses, based on the density of low lying Dirac eigenvalues in different topological sectors. The results represent one of the first measurements with dynamical overlap fermions, and they agree very well with analytic predictions.
\end{abstract}

XXIVth International Symposium on Lattice Field Theory

July 23-28, 2006

Tucson, Arizona, USA

*Speaker. 


\section{Motivation}

In 1998 a neat possibility was found to preserve chiral symmetry (in a modified form) on the lattice $[1,2,3]$. It has been used extensively in quenched QCD, but due to its computational demands the applications of dynamical chiral fermions are still in an early stage. ${ }^{1}$ Hence it is strongly motivated to develop suitable algorithmic tools, in order to arrive - within a few years at results that can be confronted with the light hadron phenomenology.

Present chiral QCD simulations are restricted to coarse lattices; in particular thermodynamic studies typically use $N_{t}=4$ and $a \approx 0.28 \mathrm{fm}$. On such lattices the standard overlap operator is non-local. Locality improves, however, if we replace the kernel by a truncated perfect hypercube operator [5]. In quenched QCD, the locality of the resulting overlap hypercube fermion (overlapHF) operator persists on rather coarse lattices [6]. At present, dynamical hypercube fermion (HF) simulations for QCD are under investigation [7]; they will indicate how far the above property still holds beyond the quenched approximation. Thermodynamic tests show already that the cutoff effects for the HF are pushed to high energy [8].

In this work, we explore the feasibility of dynamical overlap-HF simulations with an algorithm which is peculiar to this type of Ginsparg-Wilson fermions. Our testing ground is the Schwinger model (2d QED), a popular toy model with certain features similar to QCD. ${ }^{2}$ Qualitative differences from QCD are the super-renormalisability of the Schwinger model and the absence of spontaneous chiral symmetry breaking. We consider the case of two degenerate flavours.

\section{The overlap hypercube fermion}

The Ginsparg-Wilson Relation (GWR) is a criterion for a lattice modified, exact chiral symmetry [3], which was discovered by studying the properties of perfect and classically perfect [1] lattice fermions. Since those formulations involve couplings over an infinite range, a truncation is needed, which distorts the perfect symmetry and scaling properties to some extent. For the free, optimally local, perfect fermion [10] the truncation to a unit hypercube preserves excellent scaling [11] and chirality [5]. It leads to the form $D_{\mathrm{HF}, x y}=\rho_{\mu}(x-y) \gamma_{\mu}+\lambda(x-y)$, i.e. a vector term plus a scalar term ( $x, y$ are lattice sites). In $d=2$ these terms involve only couplings to nearest neighbours and across the plaquette diagonals. We gauge $D_{\mathrm{HF}}$ by multiplying the compact link variables $U_{x, \mu} \in U(1)$ along the shortest lattice paths connecting $x$ and $y$ (for the diagonal the two shortest paths are averaged) [12]. Thus we arrive at the operator $D_{\mathrm{HF}, x y}(U)$, which describes the $\mathrm{HF}^{3}$

Since $D_{\mathrm{HF}}$ is $\gamma_{5}$-Hermitian, $D_{\mathrm{HF}}^{\dagger}=\gamma_{5} D_{\mathrm{HF}} \gamma_{5}$, the exact chirality (which got lost in the truncation) can be restored by inserting $D_{\mathrm{HF}}$ into the overlap formula [2], which yields the overlap-HF operator

$$
D_{\mathrm{ovHF}}(m)=\left(1-\frac{m}{2}\right) D_{\mathrm{ovHF}}^{(0)}+m, \quad D_{\mathrm{ovHF}}^{(0)}=1+\gamma_{5} \frac{H_{\mathrm{HF}}}{\sqrt{H_{\mathrm{HF}}^{2}}}, \quad H_{\mathrm{HF}}=\gamma_{5}\left(D_{\mathrm{HF}}-1\right) .
$$

$H_{\mathrm{HF}}$ is Hermitian and $D_{\mathrm{ovHF}}^{(0)}$ fulfils the GWR in its simplest form, $\left\{D_{\mathrm{ovHF}}^{(0)}, \gamma_{5}\right\}=D_{\mathrm{ovHF}}^{(0)} \gamma_{5} D_{\mathrm{ovHF}}^{(0)} \cdot \mathrm{In}$ practice we evaluate this operator by means of Chebyshev polynomials - after projecting out the

\footnotetext{
${ }^{1}$ Recent status reports on dynamical overlap fermion simulations in QCD are given for instance in Refs. [4].

${ }^{2}$ Earliest efforts to simulate the Schwinger model with dynamical overlap fermions were reported in Ref. [9].

${ }^{3} \mathrm{We}$ are using here the HF version which is denoted as CO-HF (chirally optimised hypercube fermion) in the Ref. [12]. This is optimal for our algorithm to be described in Section 3.
} 
lowest two modes of $H_{\mathrm{HF}}^{2}$, which are treated separately. The polynomial approximation was driven to some absolute accuracy of $\varepsilon$ (see below), so we deal with $D_{\mathrm{ovHF}, \varepsilon}$.

Compared to H. Neuberger's standard overlap operator $D_{\mathrm{N}}$ [2], we replace the Wilson kernel $D_{\mathrm{W}}$ by $D_{\mathrm{HF}}$ [5]. Since the latter is an approximate solution to the GWR already, its transition $D_{\mathrm{HF}} \rightarrow D_{\mathrm{ovHF}}$ is only a modest chiral correction (in contrast to the transition $D_{\mathrm{W}} \rightarrow D_{\mathrm{N}}$ ). This property is illustrated in Fig. 1, which compares the spectra of $D_{\mathrm{HF}}$ and $D_{\mathrm{ovHF}}$ for a typical configuration at $m=0.03$ and $\beta=5$ on a $16 \times 16$ lattice. $^{4}$

Due to its perfect action background, we expect for $D_{\mathrm{HF}}$ also a good approximate rotation symmetry and scaling behaviour, which is then likely to be inherited by $D_{\text {ovHF }}$ thanks to the relation $D_{\mathrm{ovHF}} \approx D_{\mathrm{HF}}$. This relation further suggests a high level of locality for $D_{\text {ovHF, }}$ since it deviates only a little from the ultralocal operator $D_{\mathrm{HF}}$. These properties have been confirmed before in a Schwinger model study with quenched configurations, where measurement entries were re-weighted with the fermion determinant [12].

\section{A preconditioned Hybrid Monte Carlo force}

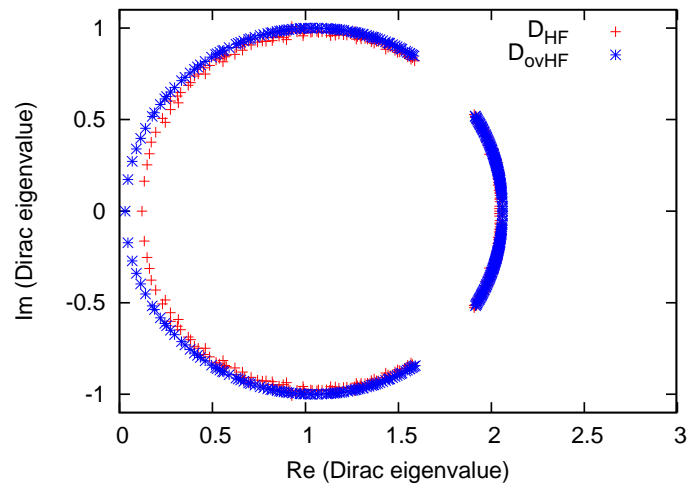

Figure 1: The spectra for $D_{\mathrm{HF}}$ and for $D_{\mathrm{ovHF}}$ (up to a gap) at $m=0.03$, in a typical, dynamical configuration at $\beta=5$ on a $16 \times 16$ lattice. Since the spectra are similar, $D_{\mathrm{HF}}$ is a good approximation to $D_{\mathrm{ovHF}}$, and therefore approximately chiral.

In order to simulate such fermions dynamically, the standard Hybrid Monte Carlo (HMC) algorithm would use the fermionic force term

$$
\bar{\psi} Q_{\mathrm{ovHF}}^{-1}\left(Q_{\mathrm{ovHF}}^{-1} \frac{\partial Q_{\mathrm{ovHF}}}{\partial A_{x, \mu}}+\frac{\partial Q_{\mathrm{ovHF}}}{\partial A_{x, \mu}} Q_{\mathrm{ovHF}}^{-1}\right) Q_{\mathrm{ovHF}}^{-1} \psi
$$

where $Q_{\mathrm{ovHF}}=\gamma_{5} D_{\mathrm{ovHF}}$ is the Hermitian overlap-HF operator, and $A_{x, \mu}$ are the non-compact gauge link variables. However, this force term is computationally expensive, and in addition conceptually problematic due to the discontinuous sign function $H_{\mathrm{HF}} / \sqrt{H_{\mathrm{HF}}^{2}}$ in $Q_{\mathrm{ovHF}}$, see eq. (2.1).

We render the force term continuous and computationally cheap by inserting only approximate overlap operators in the term (3.1). ${ }^{5}$ For the external factors we apply an overlap-HF to a low precision $\varepsilon^{\prime}$, and we use $H_{\mathrm{HF}}$ instead of $Q_{\mathrm{ovHF}}$ in the derivatives (although this could easily be extended to a low polynomial as well),

$$
\bar{\psi} Q_{\mathrm{ovHF}, \mathcal{\varepsilon}^{\prime}}^{-1}\left(Q_{\mathrm{ovHF}, \varepsilon^{\prime}}^{-1} \frac{\partial H_{\mathrm{HF}}}{\partial A_{x, \mu}}+\frac{\partial H_{\mathrm{HF}}}{\partial A_{x, \mu}} Q_{\mathrm{ovHF}, \varepsilon^{\prime}}^{-1}\right) Q_{\mathrm{ovHF}, \varepsilon^{\prime}}^{-1} \psi .
$$

The Metropolis accept/reject step is still performed with the high precision overlap operator $D_{\mathrm{ovHF}, \varepsilon}$. Hence the deviations in the force are corrected, and the only point to worry about is the acceptance

\footnotetext{
${ }^{4}$ Throughout this work we use the Wilson plaquette gauge action.

${ }^{5}$ Such a modified force might also be helpful to achieve topological transitions more frequently, but we have no data for comparison with the force (3.1).
} 
rate. Experiments with the complete simplification, which reduces $Q_{\mathrm{ovHF}, \varepsilon^{\prime}}$ to $\gamma_{5} D_{\mathrm{HF}}$, were mentioned in Ref. [13], which reported a shrinking acceptance rate for increasing volume (that work was based on the "SO-HF" of Ref. [12]). However, it turned out to be highly profitable — and still cheap - to correct the external factors to a low precision. We chose

$$
\varepsilon^{\prime}=0.005 \quad \text { (force term) }, \quad \varepsilon=10^{-16} \quad \text { (Metropolis step), }
$$

which increases the acceptance rate by an order of magnitude compared to the use of $D_{\mathrm{HF}}$ throughout the force term. Note that the force we obtain in this way is not based on a Hamiltonian dynamics, but the way we deviate from it (by proceeding from $\gamma_{5} D_{\mathrm{HF}}$ to $Q_{\mathrm{ovHF}, \varepsilon^{\prime}}$ ) does manifestly maintain the area conservation.

\section{Results for the acceptance rate, reversibility, locality and chiral condensate}

We performed production runs on a $16 \times 16$ lattice at $\beta=5$ with five masses: $m=0.03,0.06$, $0.09,0.12$ and 0.24. We applied the Sexton-Weingarten integration scheme [14] with a partial $(\delta \tau)^{3}$ error cancellation (where $\delta \tau$ is the step size). The time scales for the fermionic vs. gauge force had the ratio $1: 5$, but we did not observe a high sensitivity to this ratio. Our statistics of well thermalised configurations, separated by 200 trajectories, is given in Table 1.

Since the force (3.2) tends to push the trajectory a bit off the hyper-surface of constant energy, we kept the trajectory length (between the Metropolis steps) short. We chose it as $\ell=1 / 8$, which is divided into 20 steps (i.e. $\delta \tau=0.00625$ ); this turned out to be a good compromise in view of the acceptance rate and the dynamics between the trajectory end-points. Fig. 2 shows the acceptance rate (on the left) as well as the total number of required conjugate gradient iterations per trajectory (on the right). As usual, heavier fermions are easier to simulate. However, even down to our lightest mass of $m=0.003$ we obtained a useful acceptance rate $\approx 0.3$.
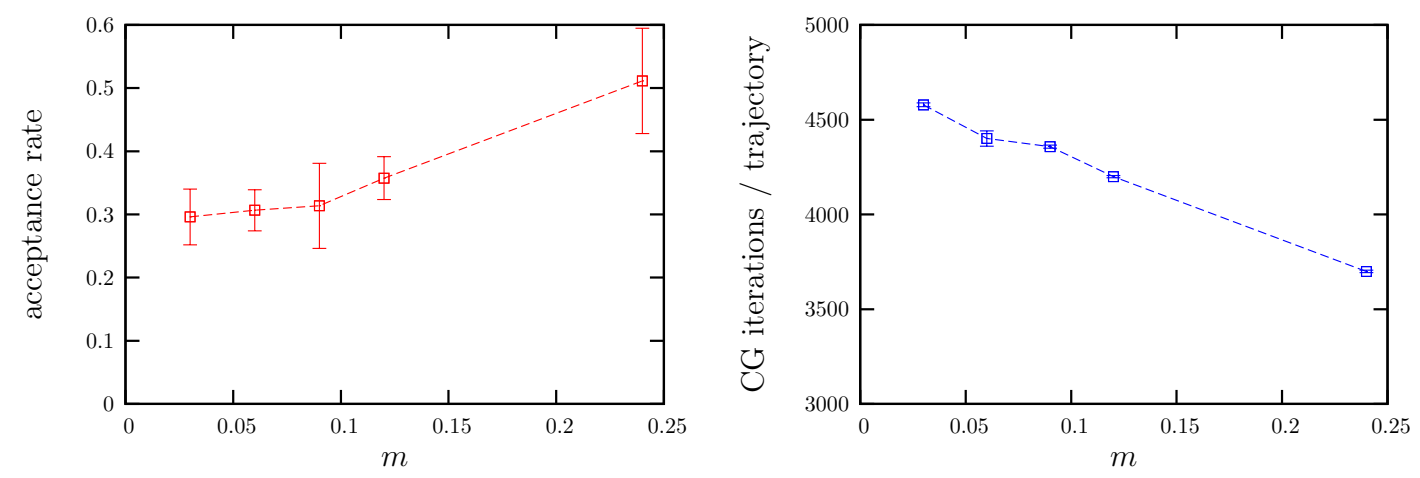

Figure 2: The acceptance rate (on the left), and the number of Conjugate Gradient iterations per trajectory (on the right, including all operations) as a function of the fermion mass $m$, on a $16 \times 16$ lattice at $\beta=5$ and trajectory length $\ell=1 / 8=20 \cdot \delta \tau$.

To study the quality of reversibility, we moved forth and back with a variable number of steps, and measured the (absolute) shift of the gauge action, $\left|\Delta S_{G}\right|$. Fig. 3 shows our results for the precision of the reversibility, still at $\delta \tau=0.00625$, for the masses $m=0.03$ and 0.12 and 0.24 . The level of reversibility seems satisfactory. As we increase the mass, it improves significantly only at $m=0.24$, as we also observed for $\delta \tau=0.005$. Our current results do not hint at any positive Lyapunov exponent, though this cannot be considered conclusive yet. 
We tested the locality in the usual way [15], by applying $D_{\mathrm{ovHF}}$ on a unit source $\eta_{y}$ and measuring the decay of the function

$f(r)=\max _{x}\left\{D_{\mathrm{ovHF}, x y} \eta_{y}\left|\sum_{\mu=1}^{2}\right| x_{\mu}-y_{\mu} \mid=r\right\}$.

We first consider the free case and demonstrate that this decay is much faster for the overlapHF operator than for the Neuberger operator, see Fig. 4 (on the left). On the right we show that the decay is still exponential for our dynamically generated configurations, which confirms the locality (and therefore the sensibility) of our Dirac operator. In the range that we considered, the mass has practically no influence on this decay rate. A previous quenched

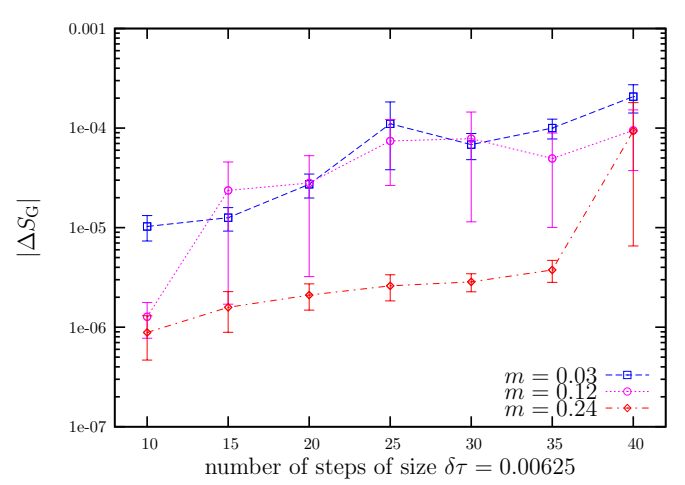

Figure 3: The reversibility precision with respect to the gauge action for a variable number of steps of length $\delta \tau=0.00625$. We show the results for our lightest mass and the two heaviest masses. We do not see an obvious indication for a positive Lyapunov exponent. re-weighted study revealed that the overlap-HF operator has a much higher degree of locality than the standard overlap operator $D_{\mathrm{N}}$ [12]. This is observed here as well, since $D_{\mathrm{ovHF}}$ at $\beta=5$ is still far more local than even the free $D_{\mathrm{N}}$. We repeat that improved locality also holds for the overlap-HF in quenched QCD [6], and it enables the installation of chiral fermions on coarser lattices than the use of $D_{\mathrm{N}}$.
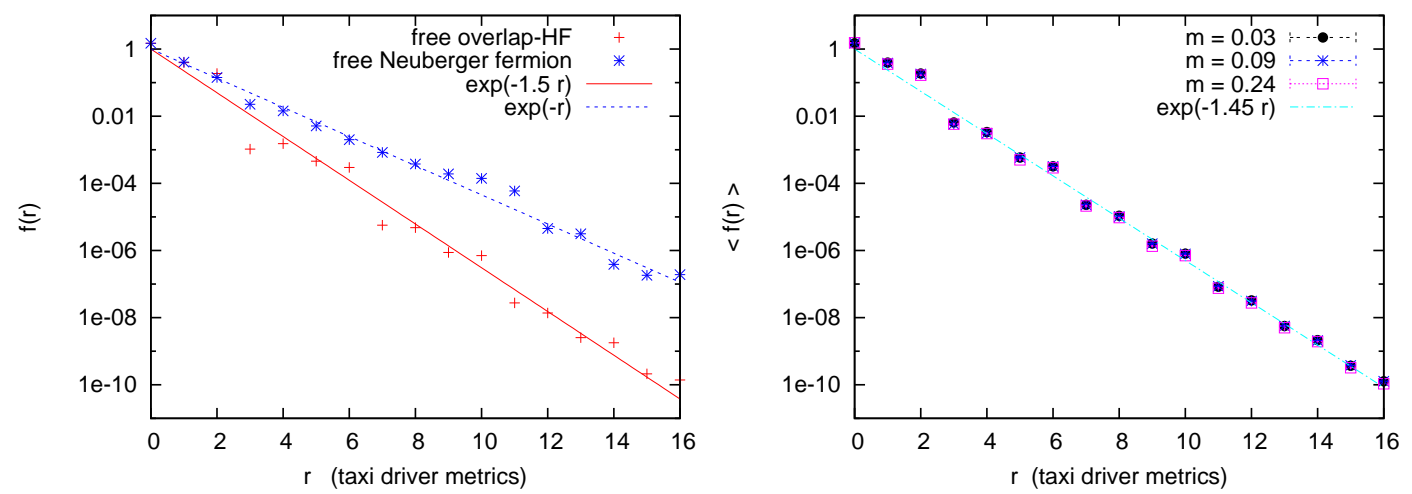

Figure 4: The locality of the overlap Dirac operators, tested by the decay of the function (4.1), against the taxi driver distance in lattice units. On the left we compare our overlap-HF operator to the standard Neuberger operator (with $H=\gamma_{5}\left(D_{\mathrm{W}}-1\right)$ ) in the free case. The plot on the right shows the exponential decay of $\langle f(r)\rangle$ based on our overlap-HF simulations with various fermion masses at $\beta=5$.

At last we address the chiral condensate; it has been studied in the 1-flavour Schwinger model with quenched configurations in Refs. [16]. For our case of two degenerate flavours, ${ }^{6}$ analytic predictions were obtained for $m \ll 1 / \sqrt{\beta}$ at low energy [18, 19]. This is realised in our settings, perhaps up to the case $m=0.24$. In particular, Ref. [19] predicts $\Sigma(m)=-\langle\bar{\psi} \psi\rangle \simeq 0.388(m / \beta)^{1 / 3}$ based on bosonisation, while Ref. [18] arrived at a slightly lower coefficient $\approx 0.37$. Both predictions are marked in Fig. 5 (below, on the right).

\footnotetext{
${ }^{6}$ For related work in QCD with dynamical overlap fermions and 1 or 2 flavours, see Refs. [17].
} 


\begin{tabular}{|c||c|c|c|c|c|}
\hline$m$ & 0.03 & 0.06 & 0.09 & 0.12 & 0.24 \\
\hline \hline \# of confs. & 205 & 235 & 221 & 458 & 100 \\
\hline \# of confs. at $v=0$ & 0 & 79 & 0 & 325 & 1 \\
\hline \# of confs. at $|v|=1$ & 205 & 156 & 220 & 133 & 46 \\
\hline \# of top. transitions & 0 & 1 & 2 & 3 & 5 \\
\hline \hline$\left\langle\lambda_{v=0}\right\rangle$ & & $0.129(3)$ & & $0.108(2)$ & \\
\hline$\left\langle\lambda_{|v|=1}\right\rangle$ & $0.171(2)$ & $0.173(2)$ & $0.171(2)$ & $0.165(3)$ & $0.169(4)$ \\
\hline$\Sigma$ & & $0.110_{-0.031}^{+0.024}$ & & $0.112_{-0.011}^{+0.014}$ & \\
\hline
\end{tabular}

Table 1: An overview of our statistics at different masses and in different topological sectors. Below we display our results for the leading non-zero eigenvalue $\lambda$ of $D_{\mathrm{ovHF}}^{(0)}$ (with jack-knife errors). The values of $\Sigma(m)$ were obtained based on the ratio between $\langle\lambda\rangle$ in the topological sectors with $|\nu|=0$ and 1 .
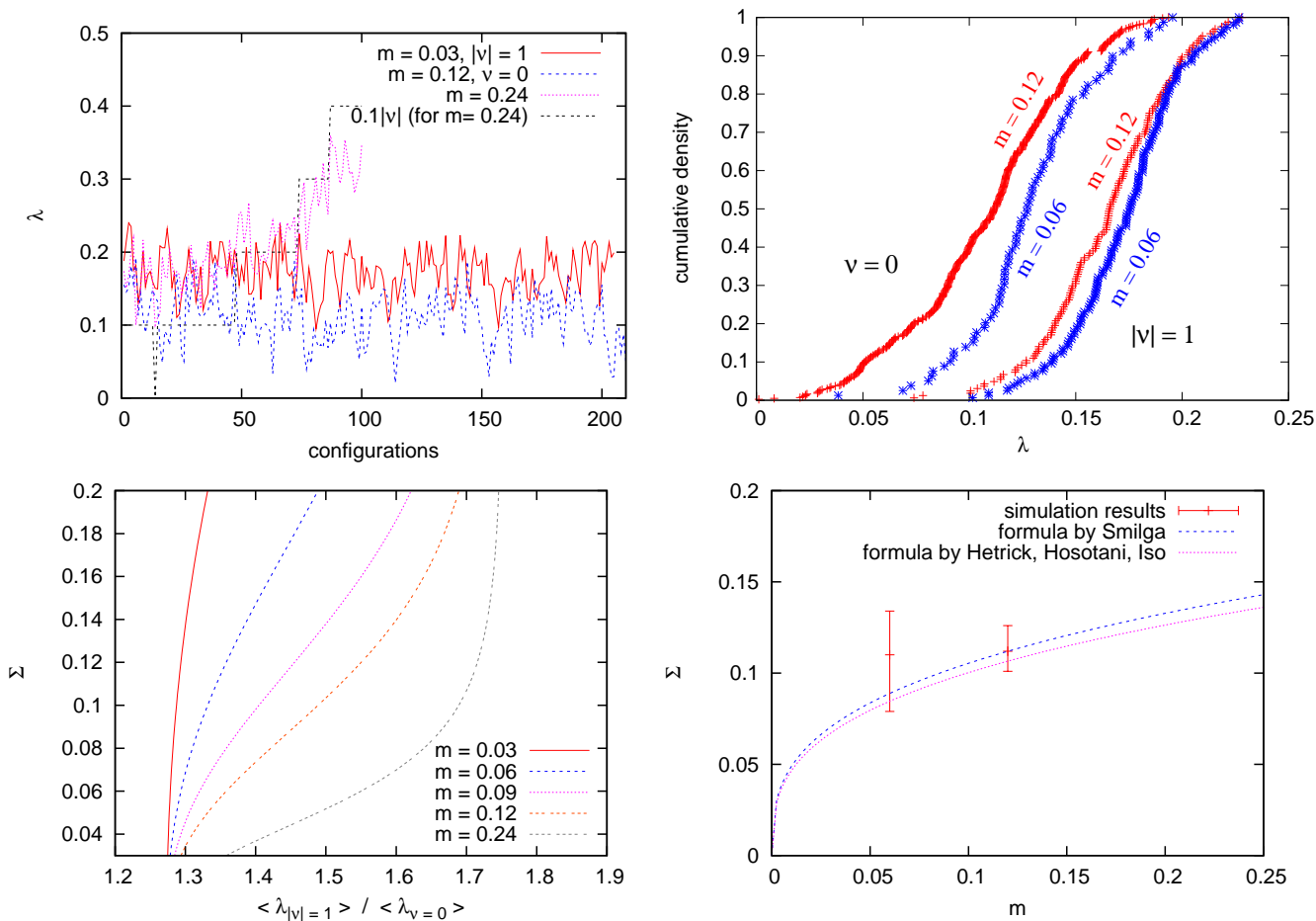

Figure 5: Above: histories (left) and cumulative densities (right) of the leading non-zero Dirac eigenvalue $\lambda$ at different masses and in different topological sectors. Below on the left: the chiral condensate $\Sigma$ as a function of the ratio $\left\langle\lambda_{|v|=1}\right\rangle /\left\langle\lambda_{v=0}\right\rangle$, according to Random Matrix Theory [21]. Below on the right we show the $\Sigma$ values that we measured at two masses, cf. Table 1, and the predictions of Refs. [18, 19].

Fig. 5 (above) shows examples for HMC histories (left, illustrating the level of de-correlation), and cumulative densities (right), of the leading non-zero Dirac eigenvalues $\lambda$. $^{7}$ For our evaluation of the chiral condensate we made use of a formula given in Ref. [21] (for the $\varepsilon$-regime), which expresses $\Sigma(m)$ as a function of the ratio between $\langle\lambda\rangle$ in the sectors with topological charge $|v|=0$ and 1. For the masses considered, these functions are plotted in Fig. 5 (below, left). We read off $\Sigma$ for the eigenvalue ratios that we measured at $m=0.06$ and $m=0.12$, see Table 1. Fig. 5 (below, right) illustrates our results, which agree with the predictions of Ref. [18, 19] within the errors.

\footnotetext{
${ }^{7} \mathrm{We}$ used the eigenvalues of $D_{\mathrm{ovHF}}^{(0)}$, stereographically projected on the imaginary axis, where we take the absolute value. This treatment worked well also in quenched QCD [20].
} 
Conclusions: We tested a force preconditioned HMC algorithm for the simulation of dynamical overlap fermions. It is applicable for the overlap-HF, but not for the standard overlap fermion, since it is designed for the case that the overlap kernel is similar to the overlap operator. In the 2-flavour Schwinger model we obtained a useful acceptance rate and a decent precision of the reversibility. A high level of locality is confirmed. We measured the chiral condensate at two fermion masses, and we obtained values for $\Sigma(m)$ consistent with analytic predictions at low energy.

Since the way to evaluate $\Sigma$ presented here leads to relatively large errors - the slopes of the functions shown in Fig. 5 (below, on the left) tend to be steep - we are now going to consider different methods for this purpose, along with an enlarged statistics.

We thank M. Hasenbusch for helpful advice, and S. Dürr, A. Kennedy and J. Verbaarschot for comments. J.V. was supported by the "Deutsche Forschungsgemeinschaft" (DFG). The computations were performed on the IBM p690 clusters of the "Norddeutscher Verbund für Hoch- und Höchstleistungsrechnen" (HLRN).

\section{References}

[1] P. Hasenfratz, V. Laliena and F. Niedermayer, Phys. Lett. B427 (1998) 125.

[2] H. Neuberger, Phys. Lett. B417 (1998) 141; Phys. Lett. B427 (1998) 353.

[3] M. Lüscher, Phys. Lett. B428 (1998) 342.

[4] N. Cundy, Nucl. Phys. (Proc. Suppl.) B153 (2006) 54. S. Schaefer, these proceedings.

[5] W. Bietenholz, Eur. Phys. J. C6 (1999) 537.

[6] W. Bietenholz, Nucl. Phys. B644 (2002) 223. S. Shcheredin, Ph.D. Thesis, Berlin (2004) [hep-lat/0502001]. W. Bietenholz and S. Shcheredin, Nucl. Phys. B754 (2006) 17.

[7] S. Shcheredin and E. Laermann, hep-lat/0609029.

[8] W. Bietenholz and U.-J. Wiese, Phys. Lett. B426 (1998) 114.

E. Laermann, C. DeTar, O. Kaczmarek and F. Karsch, Nucl. Phys. (Proc. Suppl.) 73 (1999) 447.

S. Wissel, E. Laermann, S. Shcheredin, S. Datta and F. Karsch, PoS(LAT2005)164.

[9] A. Bode, U.M. Heller, R.G. Edwards and R. Narayanan, hep-lat/9912043.

[10] W. Bietenholz and U.-J. Wiese, Nucl. Phys. B464 (1996) 319.

[11] W. Bietenholz, R. Brower, S. Chandrasekharan and U.-J. Wiese, Nucl. Phys. (Proc. Suppl.) B53 (1997) 921.

[12] W. Bietenholz and I. Hip, Nucl. Phys. B570 (2000) 423.

[13] N. Christian, K. Jansen, K.-I. Nagai and B. Pollakowski, PoS(LAT2005) 239.

[14] J.C. Sexton and D.H. Weingarten, Nucl. Phys. B380 (1992) 665.

[15] P. Hernández, K. Jansen and M. Lüscher, Nucl. Phys. B552 (1999) 363.

[16] S. Dürr and C. Hoelbling, Phys. Rev. D71 (2005) 054501.

P.H. Damgaard, U.M. Heller, R. Narayanan and B. Svetitsky, Phys. Rev. D71 (2005) 114503.

[17] T. DeGrand et al., hep-th/0605147, hep-lat/0608019.

[18] J.E. Hetrick, Y. Hosotani and S. Iso, Phys. Lett. B350 (1995) 92.

[19] A.V. Smilga, Phys. Rev. D55 (1997) 443.

[20] W. Bietenholz, K. Jansen and S. Shcheredin, JHEP 0307 (2003) 033.

[21] T. Wilke, T. Guhr and T. Wettig, Phys. Rev. D57 (1998) 6486. 